Original Article

\title{
Effects of chronic ankle instability and induced mediolateral muscular fatigue of the ankle on competitive taekwondo athletes
}

\author{
Myeounggon LeE ${ }^{1)}$, Changhong Youm, PhD $\left.^{2}\right)^{*}$, Minji Son ${ }^{1)}$, Jinhee $\mathrm{Kim}^{1)}$, \\ YOUKYUNG KIM ${ }^{1)}$ \\ 1) Biomechanics Laboratory, College of Health Sciences, Dong-A University, Republic of Korea \\ 2) Department of Health Care and Science, College of Health Sciences, Dong-A University: \\ 37 Nakdong-Daero, 550 beon-gil, Saha-gu, Busan 49315, Republic of Korea
}

\begin{abstract}
Purpose] The purpose of this study was to investigate the effects of chronic ankle instability and induced mediolateral muscular fatigue of the ankle on competitive Taekwondo athletes during single-leg drop landing. [Subjects and Methods] Fourteen competitive taekwondo athletes with chronic ankle instability and 14 healthy adults participated, and they performed three single-leg drop landings from a $40-\mathrm{cm}$ height before and after induced fatigue. Ankle angular position, peak vertical ground reaction force, loading rate, eccentric work, and contribution were calculated and analyzed. [Results] Athletes had lower ankle eversion and abduction angle than the controls did at maximum knee flexion both pre- and post-fatigue. Furthermore, athletes had lower eccentric work of the hip than the controls did post-fatigue, and they had lower eccentric work of the knee than controls at both pre- and post-fatigue. The eccentric work of the knee increased while, peak vertical ground reaction force decreased in both, athletes as well as controls post-fatigue. [Conclusion] Taekwondo athletes with chronic ankle instability who participate in a high-intensity training program are continuously exposed to potential injuries of their ankle or knee joints. Therefore, competitive taekwondo athletes with chronic ankle instability should limit their participation in regular training until they complete the rehabilitation process.

Key words: Ankle, Lateral ankle sprain, Drop landing
\end{abstract}

(This article was submitted Apr. 4, 2017, and was accepted May 9, 2017)

\section{INTRODUCTION}

Ankle sprains are one of the most common injuries in athletes ${ }^{1,2)}$. According to previous studies, up to $73 \%$ of participants with a history of ankle sprain may develop chronic ankle instability (CAI ${ }^{2}$, 3). Athletes with CAI may be susceptible to recurrent ankle sprains, loss of function, and residual symptoms such as pain, swelling, and episodes of giving way ${ }^{1-3)}$. Competitive Taekwondo (TKD) athletes perform combinations of high level kicking such as jumping, spinning, and sliding kicks during a TKD competition match ${ }^{4}$, and $98 \%$ of the score is derived from kicking in a TKD competition match ${ }^{5)}$. Particularly, the injury rate for the foot and ankle is approximately $24.1 \%$, which is higher than that for other body regions such as the head $(18.9 \%)$, thigh $(9.43 \%)$, knee $(8.42 \%)$, back $(7.63 \%)$, and leg $(6.17 \%)^{6)}$, and ankle sprains are common injury types in $\mathrm{TKD}^{7}$. The mechanism behind ankle sprain in TKD is related to either using an instep to kick or using weight-bearing on the ankle joint during stepping ${ }^{8}$. In addition, approximately $53.2 \%$ of TKD athletes with a previous ankle injury participated in a regular training session, without appropriate treatment and rehabilitation from a clinical physician ${ }^{8}$. Consequently, athletes with sustained ankle sprains may be associated with the development of $\mathrm{CAI}^{3}$. Furthermore, TKD athletes may experience fatigue while participating in a high-intensity training program or in a TKD competition match with a time limit ${ }^{8,9)}$.

*Corresponding author. Changhong Youm (E-mail: chyoum@dau.ac.kr)

(C2017 The Society of Physical Therapy Science. Published by IPEC Inc.

(c) (1) $\odot$ This is an open-access article distributed under the terms of the Creative Commons Attribution Non-Commercial No Deriva-

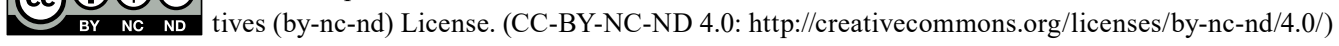


Table 1. Demographic characteristics of participants

\begin{tabular}{lcc}
\hline & CAIs $(\mathrm{n}=14)$ & Controls $(\mathrm{n}=14)$ \\
\hline Age $($ years $)$ & $20.07 \pm 0.27$ & $21.21 \pm 2.08$ \\
Height $(\mathrm{cm})$ & $176.13 \pm 9.20$ & $175.87 \pm 6.02$ \\
Weight $(\mathrm{kg})$ & $74.66 \pm 14.60$ & $73.00 \pm 7.12$ \\
BMI $\left(\mathrm{kg} / \mathrm{m}^{2}\right)$ & $23.9 \pm 2.5$ & $23.6 \pm 2.3$ \\
CAIT $(\mathrm{scores})^{\mathrm{a}}$ & $19.9 \pm 2.9$ & $28.1 \pm 1.9$ \\
Peak torque $(\mathrm{Nm} / \mathrm{kg})$ & $0.51 \pm 0.09$ & $0.48 \pm 0.09$ \\
Maximum repetitions (times) & $36.29 \pm 9.79$ & $34.50 \pm 6.30$ \\
VAS pre fatigue $(\mathrm{cm})$ & $0.68 \pm 0.8^{\mathrm{a}, \mathrm{b}}$ & $0.18 \pm 0.4^{\mathrm{a}, \mathrm{b}}$ \\
VAS post fatigue $(\mathrm{cm})$ & $3.74 \pm 1.8^{\mathrm{a}, \mathrm{b}}$ & $0.96 \pm 1.2^{\mathrm{a}, \mathrm{b}}$ \\
\hline
\end{tabular}

${ }^{a}$ The CAIs and controls were different $(\mathrm{p}<0.05)$, ${ }^{\mathrm{b}}$ Pre- and post-fatigue were different $(\mathrm{p}<0.05)$.

BMI: body mass index; CAIs: chronic ankle instability group; CAIT: Cumberland ankle instability tool; VAS: visual analog scale

The fatigue condition may reduce muscle force or power, and affects exercise or sports-related performance as well as daily activities ${ }^{10}$. A previous study reported that sports-related injuries occur at the end of an activity when the individual is fatigued, and approximately $40 \%$ of these injuries occur in the ankle joint, such as lateral ankle sprains ${ }^{11)}$. Previous studies have reported that athletes with CAI have a greater range of motion (ROM) at the ankle and knee joint than the controls do, during a single-leg drop landing ${ }^{12}$. Furthermore, Doherty et al. and De Ridder et al. reported that athletes with CAI had greater ankle inversion and displayed a stiffer landing strategy than the controls did during a single-leg drop landing ${ }^{13,14)}$. As stated above, participants with CAI demonstrated an altered landing strategy compared to controls during single-leg drop landing ${ }^{12-14)}$. This landing strategy may lead to an increase in the risk of re-sprain and episodes of giving-up on the injured-ankle ${ }^{13,14)}$.

TKD athletes require superior skills for jumping and landing abilities for TKD techniques ${ }^{15)}$, as well as the ability to balance at landing, during kicking or stepping ${ }^{16}$. However, only a few studies have focused on TKD athletes with CAI during single-leg drop landing. Furthermore, fewer studies have investigated the effects of fatigue on athletes with CAI during single-leg drop landing. Therefore, the purpose of this study was to investigate the effects of CAI and induced mediolateral muscular fatigue on the ankles of competitive TKD athletes during single-leg drop landing. We hypothesized that induced mediolateral ankle fatigue significantly affects athletes with CAI during single-leg drop landing, and these athletes may demonstrate a reduced angular position of the ankle and eccentric work done compared to controls.

\section{SUBJECTS AND METHODS}

A total of 28 participants, including 14 with unilateral CAI and 14 controls were enrolled in this study (Table 1). The following inclusion criteria were used for CAIs: (1) a male collegiate competitive TKD athlete who participated in a TKD training program for 5 days per week with a history of at least one acute lateral ankle sprain that resulted in swelling, pain, and temporary loss of function as well as, at least one episode of the ankle giving-up in the previous 6 to 24 months; (2) no other lower extremity injury within the last 6 months; (3) no history of lower extremity fracture or surgery; and (4) a score of less than 27 on the Cumberland Ankle Instability Tool (CAIT) in the ankle with CAI. Controls were volunteers involved in regular recreational activities more than twice per week. They had no history of lower extremity injury. In addition, the 2 groups were matched by age and a dominant limb (the right limb). The dominant limb was defined by self-reported answers and the task of kicking a ball ${ }^{13}$. All participants completed the CAIT before being included in the study. Participants in the control group were required to score more than 27 on the CAIT for inclusion in the study. All participants read and signed an informed consent form, approved by the Institutional Review at Dong-A university in accordance with the ethical standards of the Declaration of Helsinki (2-104709-AB-N-01-201505-HR-018-04).

During the pretest session, all participants underwent body measurements to determine each participant's body model to be used for motion capture. Height, body weight, width of the shoulder, elbow, wrist, knee, ankle, and hand thickness were measured. Leg length was measured from the anterior superior iliac spine to the medial malleolus. All participants completed the CAIT questionnaire and were scored on the visual analog scale (VAS), and ankle inversion/eversion peak torque was measured. The VAS is a simple and frequently used tool for assessment of variations in intensity of pain, with participants measuring their pain on a $10 \mathrm{~cm}$ scale. Using this scale, 0 is defined as "no pain", 1 to 3 as "mild pain", 4 to 6 as "moderate pain", and over 7 as the "worst pain"17). The VAS was scored at pre- and post-fatigue landing.

During the testing session, all participants performed pre- and post-fatigue single-leg drop landing tasks on their dominant limb. Before starting the tests, the participants performed a warm-up session incorporating 10 minutes on a cycle ergometer 
followed by static stretching. All participants were required to wear lycra shorts, and were instructed to be barefoot for the single-leg drop landing. Participants were given 26 reflective markers that were $14 \mathrm{~mm}$ in diameter, spherical in shape according to the modified Vicon's Plug-in gait full body model (Vicon, Oxford, UK), and all markers were attached to the skin, using double-sided tape and kinesiotape to secure stably. These markers were attached to the clavicle, sternum, 7th cervical vertebra, 10th thoracic vertebra, bilaterally on the front and back of the head, acromioclavicular joint, anterior superior iliac spine, posterior superior iliac spine, lateral thigh, lateral femoral epicondyle, lateral tibia, calcaneus, lateral malleolus, and second metatarsal head. To perform single-leg drop landing, participants stood on a box $40 \mathrm{~cm}$ height, with the dominant limb and were instructed to drop with the dominant limb onto the force plate located in front of the box. They performed three to five practice landings to ensure they were comfortable with the procedure. We also instructed participants to fold their upper extremities across their chest and place their non-dominant limb over the medial malleus of the dominant limb throughout the entire trial. Participants were instructed to look straight ahead. Trials were discarded if participants contacted the ground with the non-dominant foot, used the hands to maintain balance, touched the obstacle ( $1 \mathrm{~cm}$ in height) on edge of the box with their foot, or hopped or slid with the foot after landing. All participants performed three successful single-leg drop landings on the dominant limb pre- and post-fatigue.

The fatigue protocol was performed with an isokinetic dynamometer (Cybex HUMAC NORM, CSMI, USA), using a concentric-concentric protocol for inversion/eversion movement patterns at the ankle. Participants were secured with the isokinetic device in the appropriate body position, based on the Cybex calibration procedures and the isolated joint testing and exercise manual ${ }^{18}$ ). The angular velocity and range of motion for inversion/eversion were set at $60^{\circ} / \mathrm{s}$ and $50^{\circ}$ (inversion: $20^{\circ}$, eversion: $30^{\circ}$ ), respectively. Peak torque was averaged during three consecutive trials with the highest values. Termination of the fatigue protocol occurred when three consecutive repetitions below $50 \%$ of the peak torque were recorded ${ }^{11)}$. After completing the fatigue protocol, participants were detached from the dynamometer and instructed to perform the single-leg drop landing for post-testing as quickly as possible.

All data were collected and synchronized with nine infrared motion capture systems (MX-T10, Oxford Metrics, Oxford, UK) integrated with a force plate (AMTI OR6-7, Watertown, MA, USA) using the Nexus software (version 1.8.3; Vicon, UK). The sampling frequency of motion capture was set at $120 \mathrm{~Hz}$ and the GRF data was set at 1,200 Hz. A global reference system was established with the positive $\mathrm{x}$-axis as rightwards, positive $\mathrm{y}$-axis as forward, and positive $\mathrm{z}$-axis as upwards. All collected data were filtered with a second-order Butterworth low-pass filter, using a cutoff frequency of $10 \mathrm{~Hz}$, and data were analyzed after three successful landing trials ${ }^{19)}$. The events for data selection were designated as the initial contact (IC) with the force plate, when the threshold point of force plate registered more than 10 Newton, and maximum knee flexion (MKF). The angular position of the ankle was obtained at IC and MKF. Peak VGRF was normalized by body mass. Loading rate was defined as the normalized peak of VGRF divided by the time from IC to peak VGRF ${ }^{20)}$. The eccentric work done was calculated as the integral of joint power (joint moment $\times$ angular velocity) over time, where negative work (eccentric work) represented shock absorption by the joint muscle ${ }^{21)}$. Joint contribution was calculated as the percentage of the eccentric work by the particular joint over the total eccentric work of the ankle, knee and hip joints ${ }^{21)}$.

All statistical analyses were performed using SPSS for Windows (version 20.0, SPSS Inc, Chicago, IL, USA). We calculated descriptive statistics to analyze all dependent variables. The Shapiro-Wilk test was used to determine whether data had a normal distribution. All variables were compared between groups (CAIs and controls) and fatigue protocols (pre- and post-fatigue) using a two-way repeated measures, analysis of variance. Independent t-test and paired sample t-test were used to compare variables between groups and within pre- and post-fatigue for post-hoc analysis, respectively. Significance level was set at $\mathrm{p}<0.05$.

\section{RESULTS}

With respect to group differences, significant effects were observed on the angle of eversion $\left(\mathrm{F}_{1,26}=14.520, \mathrm{p}=0.001\right)$ and external rotation $\left(\mathrm{F}_{1,26}=12.463, \mathrm{p}=0.002\right)$ in the ankle at $\mathrm{MKF}$, and the eccentric work done of the $\mathrm{knee}\left(\mathrm{F}_{1,26}=9.781, \mathrm{p}=0.004\right)$ and hip joint $\left(\mathrm{F}_{1,26}=4.700, \mathrm{p}=0.040\right)$. The post-hoc analysis showed that the angle of eversion and external rotation in the ankles of athletes with CAI were significantly lower than those of controls at MKF both pre- $(\mathrm{t}=3.182$, $\mathrm{p}=0.004)$ and postfatigue ( $\mathrm{t}=3.703, \mathrm{p}=0.001)$ (Table 2). Furthermore, eccentric work done of the knee in athletes with CAI was significantly lower than that in controls both pre- $(\mathrm{t}=2.756, \mathrm{p}=0.011)$ and post-fatigue $(\mathrm{t}=3.293, \mathrm{p}=0.003)$, and the eccentric work done of hip in athletes with CAI was significantly lower than that in controls post-fatigue $(\mathrm{t}=2.197, \mathrm{p}=0.037)$ (Table 3).

With respect to induced-fatigue, significant effects were observed on the angle of plantarflexion at IC $\left(\mathrm{F}_{1,26}=7.831\right.$, $\mathrm{p}=0.012)$, dorsiflexion in the ankle at $\operatorname{MKF}\left(\mathrm{F}_{1,26}=9.393, \mathrm{p}=0.005\right)$, peak VGRF $\left(\mathrm{F}_{1,26}=17.714, \mathrm{p}=0.000\right)$, loading rate $\left(\mathrm{F}_{1,26}=18.678, \mathrm{p}=0.000\right)$, the eccentric work done of knee joint $\left(\mathrm{F}_{1,26}=29.637, \mathrm{p}=0.000\right)$, and the contribution of ankle joint $\left(\mathrm{F}_{1,26}=9.666, \mathrm{p}=0.005\right)$. Post-hoc analysis revealed that the angle of plantarflexion at post-fatigue was significantly reduced at IC in the controls $(\mathrm{t}=3.010, \mathrm{p}=0.010)$, and the angle of dorsiflexion at post-fatigue was significantly higher at MKF in the controls $(t=2.217, \mathrm{p}=0.045)$ (Table 2$)$. The peak VGRF significantly reduced at post-fatigue in both, athletes with CAI $(\mathrm{t}=2.674, \mathrm{p}=0.019)$ and controls $(\mathrm{t}=3.254, \mathrm{p}=0.006)$, and the loading rate significantly reduced at post-fatigue in the controls $(\mathrm{t}=6.429, \mathrm{p}=0.000)$. The eccentric work of the knee significantly increased at post-fatigue in both, athletes with CAI $(\mathrm{t}=2.977$, $\mathrm{p}=0.011)$ and controls $(\mathrm{t}=4.605, \mathrm{p}=0.000)$, and the contribution of ankle significantly decreased at post-fatigue in controls $(\mathrm{t}=2.525, \mathrm{p}=0.025)$ (Table 3$)$. 
Table 2. Angular position of the ankle joint

\begin{tabular}{|c|c|c|c|c|}
\hline Variable & Event & Group & Pre & Post \\
\hline \multirow{4}{*}{$\begin{array}{l}\text { Dorsi }(+) \text { / Plantar(-) flexion } \\
(\operatorname{deg})\end{array}$} & \multirow{2}{*}{ IC } & CAIs & $-26.82 \pm 4.98$ & $-24.12 \pm 4.97$ \\
\hline & & Controls $^{\mathrm{b}}$ & $-24.21 \pm 5.62$ & $-22.60 \pm 5.47$ \\
\hline & \multirow{2}{*}{ MKF } & CAIs & $26.01 \pm 3.65$ & $29.48 \pm 6.05$ \\
\hline & & Controls $^{\mathrm{b}}$ & $30.20 \pm 7.43$ & $33.19 \pm 7.13$ \\
\hline \multirow{4}{*}{$\begin{array}{l}\text { Eversion }(+) / \text { Inversion }(-) \\
(\mathrm{deg})\end{array}$} & \multirow{2}{*}{ IC } & CAIs & $-1.06 \pm 1.98$ & $-1.27 \pm 1.66$ \\
\hline & & Controls & $-0.17 \pm 3.05$ & $0.22 \pm 2.84$ \\
\hline & \multirow{2}{*}{$\mathrm{MKF}^{\mathrm{a}}$} & CAIs & $1.84 \pm 1.81$ & $2.34 \pm 2.00$ \\
\hline & & Controls & $4.30 \pm 2.26$ & $5.29 \pm 2.21$ \\
\hline \multirow{4}{*}{$\begin{array}{l}\text { Adduction }(+) / \text { Abduction }(-) \\
(\text { deg) }\end{array}$} & \multirow{2}{*}{ IC } & CAIs & $5.60 \pm 11.28$ & $6.28 \pm 12.02$ \\
\hline & & Controls & $-3.39 \pm 13.32$ & $-4.08 \pm 14.11$ \\
\hline & \multirow{2}{*}{$\mathrm{MKF}^{\mathrm{a}}$} & CAIs & $-14.09 \pm 10.29$ & $-12.70 \pm 10.55$ \\
\hline & & Controls & $-24.52 \pm 9.39$ & $-27.29 \pm 9.05$ \\
\hline
\end{tabular}

${ }^{a}$ Independent $\mathrm{t}$-test between groups $(\mathrm{p}<0.05)$, ${ }^{\text {PPaired }} \mathrm{t}$-test between pre and post fatigue $(\mathrm{p}<0.05)$.

CAIs: chronic ankle instability group; IC: initial contact; MKF: maximum knee flexion

Table 3. Peak VGRF, loading rate, and eccentric work and contribution of ankle, knee, and hip

\begin{tabular}{llcc}
\hline Variable & Group & Pre & Post \\
\hline Peak VGRF & CAIs $^{\mathrm{b}}$ & $27.39 \pm 1.87$ & $26.67 \pm 1.63$ \\
$(\mathrm{~N} / \mathrm{kg})$ & Controls $^{\mathrm{b}}$ & $27.27 \pm 1.49$ & $26.31 \pm 2.06$ \\
Loading rate & CAIs & $284.55 \pm 31.52$ & $274.48 \pm 27.86$ \\
$(\mathrm{~N} / \mathrm{kg} / \mathrm{s})$ & Controls $^{\mathrm{b}}$ & $306.63 \pm 22.86$ & $283.48 \pm 28.33$ \\
& CAIs & $-1.15 \pm 0.22$ & $-1.13 \pm 0.16$ \\
Ankle $(\mathrm{J} / \mathrm{kg})$ & Controls & $-1.23 \pm 0.20$ & $-1.21 \pm 0.23$ \\
& CAIs & $-1.58 \pm 0.38^{\mathrm{a}}$ & $-1.71 \pm 0.37^{\mathrm{a}}$ \\
Knee $(\mathrm{J} / \mathrm{kg})$ & Controls & $-1.94 \pm 0.32^{\mathrm{a}}$ & $-2.18 \pm 0.39^{\mathrm{a}}$ \\
& CAIs & $-0.84 \pm 0.35$ & $-0.89 \pm 0.38^{\mathrm{a}}$ \\
Hip $(\mathrm{J} / \mathrm{kg})$ & Controls & $-1.11 \pm 0.37$ & $-1.22 \pm 0.42^{\mathrm{a}}$ \\
& CAIs & $32.78 \pm 6.74$ & $29.00 \pm 4.96$ \\
Ankle $(\%)$ & Controls ${ }^{\mathrm{b}}$ & $31.00 \pm 5.92$ & $26.60 \pm 5.56$ \\
Knee $(\%)$ & CAIs & $44.12 \pm 7.40$ & $45.82 \pm 6.55$ \\
& Controls & $45.82 \pm 6.55$ & $47.40 \pm 6.39$ \\
Hip $(\%)$ & CAIs & $23.10 \pm 6.78$ & $23.20 \pm 7.32$ \\
& Controls & $25.50 \pm 5.92$ & $26.00 \pm 6.52$ \\
\hline
\end{tabular}

andependent $\mathrm{t}$-test between groups $(\mathrm{p}<0.05)$, bPaired t-test between pre and post fatigue $(\mathrm{p}<0.05)$.

CAIs: chronic ankle instability group; VGRF: vertical ground reaction force

\section{DISCUSSION}

We hypothesized that induced mediolateral ankle fatigue would significantly affect athletes with CAI during single-leg drop landing, demonstrating a decrease in angular position of the ankle and eccentric work done, as compared to controls.

In this study, athletes with CAI exhibited a lower angle of eversion and abduction in the ankle joint than the controls did, pre- and post-fatigue at MKF. Kernozek et al. reported that women have a greater angle of dorsiflexion and eversion in the ankle than men $\mathrm{do}^{22}$. Furthermore, they suggested that an increased angle of dorsiflexion and eversion in the ankle joint may be associated with the shock absorption strategy, which may reduce VGRF and delay its peak by producing a greater ankle ROM. Therefore, we suggest that athletes with CAI may have reduced shock absorption capacity adjusted ankle joint angle compared to controls during single-leg drop landing.

The present study showed that athletes with CAI displayed lower eccentric work of the knee than the controls did pre- and post-fatigue. A greater angle of dorsiflexion and knee flexion may dissipate an impact force during single-leg drop landing $^{22,23)}$. However, the CAI participants with neuromuscular impairments caused by lateral sprains may rely on inappropriate 
coordination strategies to dissipate the impact force by restricting movement of the joints of the lower limbs during single-leg drop landing ${ }^{12-14,23)}$. Furthermore, Terada et al. reported that athletes with CAI have a higher contribution of the ankle and lower contribution of the knee than the controls during a stop-jump task; these contributions were $54.3 \%, 18.2 \%$, and $27.5 \%$ in the CAI group, and $40.9 \%, 27.3 \%$, and $31.9 \%$ in the controls, representing the ankle, knee, and hip, respectively ${ }^{23)}$. In this study, both athletes with CAI and controls had a higher dependence on the knee during single-leg drop landing regardless of the induced fatigue (Table 3). However, the CAI group in our study had a higher dependence on the ankle than controls in post-fatigue, even though it was not significant difference $(\mathrm{p}=0.053)$, which may increase their risk of recurrent ankle injury. In addition, Terada et al. reported a higher dependence on the ankle, while in the current study there was a higher dependence on the knee ${ }^{23)}$. These inconsistencies may be associated with a difference in the tasks of stop-jump landing and single-leg drop landing. Furthermore, Zhang et al. reported that contributions of the ankle, knee, and hip joint to shock absorption during drop landing from a $32 \mathrm{~cm}$ height were $21 \%, 47 \%$, and $32 \%$ respectively ${ }^{24}$, and the largest contributor of shock absorption was the knee joint, consistent with this study. These results were also similar with the healthy college basketball players $^{25)}$ and recreational sports participants ${ }^{26-28)}$ that the knee joint had greater eccentric work and contribution than the hip and ankle joint, and the greater knee extensors strength may influence to increase the eccentric work done of knee joint during landing ${ }^{27}$ ). Therefore, the greater knee extensors may be a main factor to absorb the impact force and reduce the injury risk during landing tasks ${ }^{21,29)}$.

However, as reported by Gribble and Robinson, participants with CAI exhibited lower force production of the knee extensors compared to healthy controls using an isokinetic device ${ }^{30)}$. Further, this restricted knee extensors may be affected by $\mathrm{CAI}^{30}$. Although we did not examine the knee extensors moment, the greater eccentric work may be influenced by the increased knee extension moment due to the eccentric work was calculated by integral of joint power (joint moment $\times$ angular velocity) over time. Therefore, as shown by the results of present study, the lower eccentric work of the knee in athletes with CAI may be related to deficits in force production of the knee extensors. Furthermore, approximately $52 \%$ to $60 \%$ of participants with ACL injury have a history of lateral ankle sprain ${ }^{31)}$. This may be because of altered neuromuscular control of the knee and hip joint caused by the lateral ankle sprain, which may increase the potential risk of ACL injury in athletes with $\mathrm{CAI}^{31)}$. Therefore, a prevention program for ACL injury should specifically be considered as an addition to an ankle rehabilitation program ${ }^{31)}$. The CAI group in the present study consisted of competitive TKD athletes who participated in a TKD training program regularly for 5 days per week. If these athletes participated in a high-intensity training program continuously, they would potentially be exposed to injury of their ankle or knee joint ${ }^{30,31)}$. Therefore, we suggest that competitive TKD athletes with CAI rehabilitate their ankle joint completely to reduce the potential risks of injury.

The peak VGRF was reduced in both the athletes with CAI and the controls after fatigue protocols, which was consistent with the study by Madigan and Pidcoe ${ }^{20}$. They reported that peak VGRF was significantly reduced after the fatigue protocol during single-leg landing, and that the landing biomechanics that were altered by fatigue protocols may be associated with a neuromuscular protective mechanism to decrease impact force. It is possible that the increased angle of dorsiflexion and the reduced peak VGRF after fatigue protocols will reduce the impact force on lower limbs during single-leg drop landing, which may reduce the potential risks of ACL injury to the lower limbs ${ }^{32}$. However, the CAI group in our study showed a reduced peak VGRF after the fatigue protocol, whereas the angle of dorsiflexion was not significantly altered $(p=0.052)$. Therefore, future studies including a larger sample size of participants with CAI, may help in understanding the impact of angle of dorsiflexion during single-leg drop landing.

Moreover, our results show that the loading rate was significantly reduced in controls after the fatigue protocol, but not in the athletes with CAI. The high loading rate may be associated with the increased VGRF and reduced lower limb neuromuscular function during landing tasks ${ }^{33}$. De Ridder et al. reported a negative correlation between the ROM of ankle and the loading rate, and a smaller ROM of the ankle with a higher loading rate may increase the risks of ankle injury, including episodes of giving-up and occurrence of ankle sprains in athletes with CAI during single-leg drop landing ${ }^{14)}$. One of the strategies reported to reduce ankle injury risks during single-leg drop landing is to perform less plantarflexion at IC and greater dorsiflexion at the landing phase ${ }^{12,14,22,32)}$. The controls in the present study showed a reduced angle of plantarflexion at IC after the fatigue protocol, which may be associated with a protective strategy to prevent re-strain on the ankle. Furthermore, the angle of dorsiflexion was increased at MKF, and the peak VGRF was reduced after the fatigue protocol, which may be a strategy to decrease the loading rate for preventing injury risks. The athletes with CAI showed no significant change in the loading rate after the fatigue protocol, which may be due to a lack of significant changes in the angles of plantarflexion and dorsiflexion in the ankle when comparing pre- and post-fatigue. The participants with CAI sustained impairments in neuromuscular control, which may contribute to inappropriate coordination strategies during single-leg drop landing ${ }^{12-14)}$. Therefore, we suggest that participants with CAI have a reduced capacity to control the impact force due to inadequate landing kinematic strategies after induced fatigue, which may increase their risks of potential injury in the ankle joint.

In the present study, the eccentric work of the knee was increased in both the CAI group and the controls after the fatigue protocol. Kim and Youm ${ }^{34)}$ reported that the eccentric work of the knee was reduced and the ankle joint was the largest contributor to shock absorption in both overweight and control groups after a fatigue protocol. Coventry et al. reported that after a fatigue protocol, the eccentric work of the hip increased, while that of the ankle decreased, and there was no significant difference in the knee eccentric work ${ }^{29}$. This inconsistency among previous studies and ours may be a result of differences in fatigue protocols. In the current study, the contribution of the knee was increased in both the CAI group (1.7\%) 
and the controls (1.9\%) after the mediolateral ankle fatigue protocol. These results indicate a strategy used to increase the shock absorption ability using the knee extensor muscles during single-leg drop landing in athletes with CAI and controls. Furthermore, the high-level performances in TKD require challenging jumping and landing techniques ${ }^{15}$, which may contribute to an increase in load on the knee joint and higher risks of ACL injury during the landing phase after performances ${ }^{16)}$. If the knee contribution had increased by muscle fatigue, then the potential injury risks would increase in all participants with CAI during TKD training programs or matches. Therefore, we suggest that competitive TKD athletes with CAI may be exposed to the possibility of injury at the knee joint during fatigue conditions, and thus, they should limit their participation in regular training until they have completed the rehabilitation process for their ankle joint. This information may be useful for clinicians in the rehabilitation of patients with ankle sprains and CAI.

There are several limitations to this study. First, we recruited participants based on the prior power analysis, but our sample size may be small to determine significant group differences. Second, we could not recruit healthy TKD athletes who did not have CAI. Most competitive TKD athletes sustained a lower limb injury within the last 6 months or had history of lower extremity fracture or surgery, making it difficult to recruit healthy TKD athletes. Third, all recruited participants were men. Future studies need to evaluate the gender differences in TKD athletes with CAI. Fourth, we only used a $40 \mathrm{~cm}$ box for the single-leg drop landing exercise. Fifth, the mediolateral ankle fatigue was induced by an isokinetic dynamometer, and the angular velocity was set at $60 \%$ s. The protocol for angular velocity is categorized into slow (i.e., $60 \%$ s) and fast (i.e., $180^{\circ}$ s), which may result in different effects of fatigue. Finally, we did not consider muscle activation of the lower limbs during single-leg drop landing.

In summary, the CAI demonstrated decreased the shock absorption strategies compared to the controls, and induced mediolateral ankle fatigue affected the capacity to control the impact force by inadequate landing kinematic strategies. It is possible that TKD athletes with CAI who participate in a high-intensity training program continuously could be exposed to potential injury of their ankle or knee joint. Therefore, we suggest that TKD competitive athletes with CAI should limit participation in regular training until they have completed the rehabilitation process.

\section{REFERENCES}

1) Hoch MC, Farwell KE, Gaven SL, et al.: Weight-bearing dorsiflexion range of motion and landing biomechanics in individuals with chronic ankle instability. J Athl Train, 2015, 50: 833-839. [Medline] [CrossRef]

2) Terada M, Pietrosimone B, Gribble PA: Individuals with chronic ankle instability exhibit altered landing knee kinematics: potential link with the mechanism of loading for the anterior cruciate ligament. Clin Biomech (Bristol, Avon), 2014, 29: 1125-1130. [Medline] [CrossRef]

3) Delahunt E, Coughlan GF, Caulfield B, et al.: Inclusion criteria when investigating insufficiencies in chronic ankle instability. Med Sci Sports Exerc, 2010, 42: 2106-2121. [Medline] [CrossRef]

4) Lystad RP, Pollard H, Graham PL: Epidemiology of injuries in competition taekwondo: a meta-analysis of observational studies. J Sci Med Sport, 2009, 12: 614-621. [Medline] [CrossRef]

5) Jakubiak N, Saunders DH: The feasibility and efficacy of elastic resistance training for improving the velocity of the Olympic Taekwondo turning kick. J Strength Cond Res, 2008, 22: 1194-1197. [Medline] [CrossRef]

6) Kazemi M, Chudolinski A, Turgeon M, et al.: Nine year longitudinal retrospective study of Taekwondo injuries. J Can Chiropr Assoc, 2009 , 53: $272-281$. [Medline]

7) Varkiani ME, Alizadeh MH, Kazemi M, et al.: Taekwondo competition injuries in Iranian premier league: a prospective study. Int J Sport Stud, 2013, 3: $542-548$.

8) Kim WW, Cho KS: The effects of board training and complex training on ankle stability in taekwondo students with a history of ankle sprain. J Muscle Jt Health, 2011, 18: 182-191. [CrossRef]

9) Jeong SK, Ham WT: The difference of body composition, exercise ability, and fatigue factors by the maximal aerobic exercise in Taekwondo sparing players. Korea J Sports Sci, 2008, 17: 497-505.

10) Boyas S, Remaud A, Bisson EJ, et al.: Impairment in postural control is greater when ankle plantarflexors and dorsiflexors are fatigued simultaneously than when fatigued separately. Gait Posture, 2011, 34: 254-259. [Medline] [CrossRef]

11) Yaggie JA, McGregor SJ: Effects of isokinetic ankle fatigue on the maintenance of balance and postural limits. Arch Phys Med Rehabil, 2002, 83: 224-228. [Medline] [CrossRef]

12) Caulfield BM, Garrett M: Functional instability of the ankle: differences in patterns of ankle and knee movement prior to and post landing in a single leg jump. Int J Sports Med, 2002, 23: 64-68. [Medline] [CrossRef]

13) Doherty C, Bleakley C, Hertel J, et al.: Single-leg drop landing motor control strategies following acute ankle sprain injury. Scand J Med Sci Sports, 2015, 25: 525-533. [Medline] [CrossRef]

14) De Ridder R, Willems T, Vanrenterghem J, et al.: Multi-segment foot landing kinematics in subjects with chronic ankle instability. Clin Biomech (Bristol, Avon), 2015, 30: 585-592. [Medline] [CrossRef]

15) Park JK: Effects of blindfolded eyes on ground reaction forces of elementary school taekwondo trainees and non-trainees in drop landing. Taekwondo J Kukkiwon, 2011, 2: 51-60. [CrossRef]

16) Yoon SD, Sung DH, Park GD: The effect of active core exercise on fitness and foot pressure in Taekwondo club students. J Phys Ther Sci, 2015, 27: 509-511. [Medline] [CrossRef]

17) Hawker GA, Mian S, Kendzerska T, et al.: Measures of adult pain: visual analog scale for pain (vas pain), numeric rating scale for pain (nrs pain), mcgill pain 
questionnaire (mpq), short-form mcgill pain questionnaire (sf-mpq), chronic pain grade scale (cpgs), short form-36 bodily pain scale (sf-36 bps), and measure of intermittent and constant osteoarthritis pain (icoap). Arthritis Care Res (Hoboken), 2011, 63: S240-S252. [Medline] [CrossRef]

18) Jerosch J, Hoffstetter I, Bork H, et al.: The influence of orthoses on the proprioception of the ankle joint. Knee Surg Sports Traumatol Arthrosc, 1995, 3: 39-46. [Medline] [CrossRef]

19) Gehring D, Melnyk M, Gollhofer A: Gender and fatigue have influence on knee joint control strategies during landing. Clin Biomech (Bristol, Avon), 2009, 24: 82-87. [Medline] [CrossRef]

20) Madigan ML, Pidcoe PE: Changes in landing biomechanics during a fatiguing landing activity. J Electromyogr Kinesiol, 2003, 13: 491-498. [Medline] [CrossRef]

21) Yeow CH, Lee PV, Goh JC: Effect of landing height on frontal plane kinematics, kinetics and energy dissipation at lower extremity joints. J Biomech, 2009, 42: 1967-1973. [Medline] [CrossRef]

22) Kernozek TW, Torry MR, VAN Hoof H, et al.: Gender differences in frontal and sagittal plane biomechanics during drop landings. Med Sci Sports Exerc, 2005, 37: 1003-1012, discussion 1013. [Medline]

23) Terada M, Pfile KR, Pietrosimone BG, et al.: Effects of chronic ankle instability on energy dissipation in the lower extremity. Med Sci Sports Exerc, 2013, 45: 2120-2128. [Medline] [CrossRef]

24) Zhang SN, Bates BT, Dufek JS: Contributions of lower extremity joints to energy dissipation during landings. Med Sci Sports Exerc, 2000, 32: 812-819. [Medline] [CrossRef]

25) Zhang S, Clowers K, Kohstall C, et al.: Effects of various midsole densities of basketball shoes on impact attenuation during landing activities. J Appl Biomech, 2005, 21: 3-17. [Medline] [CrossRef]

26) Gardner JK, McCaw ST, Laudner KG, et al.: Effect of ankle braces on lower extremity joint energetics in single-leg landings. Med Sci Sports Exerc, 2012, 44: 1116-1122. [Medline] [CrossRef]

27) Montgomery MM, Shultz SJ, Schmitz RJ, et al.: Influence of lean body mass and strength on landing energetics. Med Sci Sports Exerc, 2012 , $44: 2376-2383$. [Medline] [CrossRef]

28) Zhang S, Derrick TR, Evans W, et al.: Shock and impact reduction in moderate and strenuous landing activities. Sports Biomech, 2008, 7: 296-309. [Medline] [CrossRef]

29) Coventry E, O'Connor KM, Hart BA, et al.: The effect of lower extremity fatigue on shock attenuation during single-leg landing. Clin Biomech (Bristol, Avon), 2006, 21: 1090-1097. [Medline] [CrossRef]

30) Gribble PA, Robinson RH: An examination of ankle, knee, and hip torque production in individuals with chronic ankle instability. J Strength Cond Res, 2009, 23: 395-400. [Medline] [CrossRef]

31) Kramer LC, Denegar CR, Buckley WE, et al.: Factors associated with anterior cruciate ligament injury: history in female athletes. J Sports Med Phys Fitness, 2007, 47: 446-454. [Medline]

32) Fong CM, Blackburn JT, Norcross MF, et al.: Ankle-dorsiflexion range of motion and landing biomechanics. J Athl Train, 2011, 46: 5-10. [Medline] [CrossRef]

33) Quatman CE, Ford KR, Myer GD, et al.: Maturation leads to gender differences in landing force and vertical jump performance: a longitudinal study. Am J Sports Med, 2006, 34: 806-813. [Medline] [CrossRef]

34) Kim TH, Youm CH: Effects of knee joint muscle fatigue and overweight on shock absorption during single-leg landing of adult women. Kor J Sport Biomech, 2014, 24: 59-66. [CrossRef] 